\title{
Citología vaginal y factores de riesgo para cáncer de cérvix en adolescentes escolares de Manizales: conocimientos, actitudes y exposición
}

\author{
Germán Arango R.*; Martha Inés Urrego O.**; \\ María Teresa Vallejo G.; Martha Lucía Vanegas P.***; Diego Zuluaga M.****
}

\begin{abstract}
RESUMEN: Este estudio descriptivo-relacional identificó la actitud y el conocimiento hacia la citología vaginal, factores de riesgo y exposición para cáncer de cuello uterino en las adolescentes escolares de Manizales.

De 5.943 adolescentes, se seleccionaron 37.4 de los grados 9o., 10o. y 110., a las cuales se les aplicó una encuesta, escala de actitudes y prueba de conocimientos. Al total de la población (10.305) se realizó una labor educativa sobre diferentes aspectos de esta problemática. Hubo buen conocimiento sobre factores de riesgo y citología vaginal.
\end{abstract}

El $46.7 \%$ tuvo una actitud indiferente hacia esta última. El 30.6\% está expuesta a los factores de riesgo. El $38 \%$ ha tenido relaciones sexuales, de las cuales sólo el $5.5 \%$ se ha sometido a una citología y el $69 \%$ las inició de los 13 a los 18 años.

Los métodos anticonceptivos son empleados por el $51.4 \%$.

De los factores de riesgo, los de menor conocimiento para las adolescentes fueron "edad del primer coito" y "embarazo antes de los 18 años".

Las adolescentes de mayor exposición fueron de 17 años y las pertenecientes a colegios mixtos y públicos.

PALABRAS CLAVES: Citología vaginal, factores de riesgo, actitud, cáncer de cérvix, adolescente.

SUMMARY: This descriptive-relational study was designed to identify knowledge and attitude regarding vaginal cytology, risk factors and exposition to them among scholar adolescent females in Manizales.

We carried out a poll, administered an attitude scale and a knowledge test, to a sample of 37.4 students (selected from 5.943 adolescent females).

$46.7 \%$ showed indifferent attitude regard to vaginal cytology.

$30.6 \%$ were exposed to risk factors.

$38.0 \%$ had had sexual experience, and of these only $5.5 \%$ had undergone one cytologic smear. $69 \%$ started sexual activities between 13 and 18 years.

$51.4 \%$ used contraceptive methods.

The least known risk factors among adolescents were "age at first intercourse" and "pregnancy before 18 years".

The most exposed adolescents were 17 years old and belonged to mixed and public schools.

KEY WORDS: Vaginal cytology, risk factors, cervix, cancer, adolescent, attitude.

\section{Introducción}

Colombia tiene la incidencia más alta de cáncer de cérvix en el mundo, lo que aumenta considerablemente los costos de búsqueda, diagnóstico y hospitalización de las pacientes con esta enfermedad, afectando el presupuesto destinado a los programas de salud (1).

\footnotetext{
* Médico Gineco-Obstetra y Oncólogo. Profesor Asistente. Facultad de Medicina, Universidad de Caldas, Manizales.

** Enfermera, Profesora Titular, Facultad de Enfermería. Universidad de Caldas, Manizales.
}

Un gran porcentaje de mujeres en la fase más activa de sus vidas, reúne las condiciones propicias para adquirir esta neoplasia. El $70 \%$ de las pacientes consultan en estadios avanzados, ya que los programas sobre detección precoz del cáncer de cérvix tanto a nivel de educación como de oferta de servicios, no son suficientes para cubrir grandes áreas de la población.

\footnotetext{
*** Enfermera, Profesora Asociada, Facultad de Enfermería. Universidad de Caldas, Manizales.

**** Psicólogo. Profesor Asociado. Facultad de Medicina. Universidad de Caldas, Manizales.
} 
En Caldas existen 225.000 mujeres en riesgo de adquirir carcinoma del cuello uterino y solamente se realizan 30.000 citologías anuales, observándose que el 90\% de la población queda sin acceso a este recurso (2).

A través de los tiempos se ha observado una asociación directa entre el comportamiento sexual y la aparición de algunas patologías que han hecho eco en diferentes décadas de la historia y actualmente el cáncer del cuello uterino ha demostrado que reúne las características epidemiológicas para clasificarla como enfermedad de transmisión sexual (3-4).

Para lograr un diagnóstico adecuado y poder orientar el tratamiento respectivo, es necesario aplicar los medios diagnósticos de una manera coordinada, teniendo en cuenta que la colposcopia y la citología se complementan y si se canalizan los recursos según los factores de riesgo, se obtendrá una detección del cáncer del cuello uterino, en sus etapas preinvasoras, las cuales son curables en el $100 \%$ de los casos.

La colposcopia es el mé todo ideal para obtener biopsias dirigidas de las imágenes sospechosas en la zona de transformación del cuello uterino y se debería aplicar a todas las pacientes dentro de la consulta ginecológica rutinaria (16). Pero debido a que se considera un procedimiento especializado y de un entrenamiento adecuado por parte del médico, no es posible utilizarlo como método masivo en la detección precoz de cáncer de cuello uterino y su uso está restringido en aquellas pacientes cuya citología vaginal ha sido reportada como positiva para neoplasia.

Es por esto que se debe racionalizar el recurso y utilizarse de acuerdo con los factores de riesgo para adquirir esta patología y que de acuerdo con varios autores, se han logrado identificar los siguientes: Inicio temprano de la actividad sexual, promiscuidad sexual tanto masculina como femenina, primer embarazo antes de los 18 años, tabaquismo, no uso de preservativo, enfermedades de transmisión sexual incluyendo el Virus del Papiloma Humano (5-7).

\section{Materiales y métodos}

El estudio realizado fue Descriptivo-Relacional. Para la obtención de la muestra se empleó la técnica estadística de Muestra aleatoria de proporciones en forma estratificada.

Se seleccionaron 374 adolescentes mujeres de los grados $90 ., 100$. y 110. , pertenecientes a 57 colegios mixtos, femeninos, públicos y privados de una población adolescente femenina de 5.943, a las cuales se les aplicó los siguientes instrumentos:

a) Escala de actitudes, para evaluar la disposición de las adolescentes hacia la citología vaginal, en tres niveles (positiva, indiferente y negativa).

b) Prueba de conocimientos, para obtener el nivel de información que tienen las adolescentes acerca de la citología vaginal y los factores de riesgo para adquirir cáncer de cuello uterino.

c) Encuesta, a través de la cual se registraron algunos elementos socio-demográficos del encuestado y la expo- sición del mismo a los factores de riesgo para adquirir cáncer de cuello uterino.

En los 57 colegios, se impartió educación a 10.305 adolescentes de ambos sexos sobre esta temática, por medio de una conferencia con sus respectivas diapositivas elaboradas por los autores. Para complementar esta información, se distribuyó un plegable a cada uno de los asistentes sobre esta temática.

Para poder realizar estas actividades, fue necesario contactar la Secretaría de Educación de Caldas y del Municipio de Manizales, para coordinar un encuentro con los Rectores y Psicoorientadores de las Instituciones Educativas del nivel de educación básica secundaria y media vocacional de la ciudad de Manizales, a quienes se les realizó un Seminario-Taller sobre la temática, con el fin de motivarlos y lograr su cooperación para la presente investigación.

\section{Resultados}

De acuerdo con los objetivos propuestos en el estudio, se presentan a continuación los resultados generados del mismo.

\section{Tabla 1 \\ CONOCIMIENTO SOBRE CITOLOGIA VAGINAL Y FACTORES DE RIESGO PARA CANCER DE CERVIX EN ADOLESCENTES ESCOLARES DE MANIZALES}

\begin{tabular}{|lrcrc|}
\hline \multicolumn{3}{rc|}{ Factores de riesgo } & \multicolumn{2}{c|}{ Citología vaginal } \\
\hline Nivel & $\mathrm{N}$ & $\%$ & $\mathrm{~N}$ & $\%$ \\
Excelente & 76 & 20.3 & 82 & 22 \\
Bueno & 255 & 68.2 & 255 & 68.2 \\
Regular & 23 & 6.1 & 25 & 6.7 \\
Deficiente & 20 & 5.4 & 12 & 3.1 \\
\hline Total & 374 & 100 & 374 & 100 \\
\hline
\end{tabular}

Como se puede observar, las adolescentes escolares de Manizales tienen un grado de conocimiento bueno acerca de la citología vaginal y de los factores de riesgo para adquirir cáncer de cuello uterino.

Figura 1

EXPOSICION A LOS FACTORES DE RIESGO

PARA ADQUIRIR CANCER DE CUELLO UTERINO EN LAS ADOLESCENTES ESCOLARES DE MANIZALES

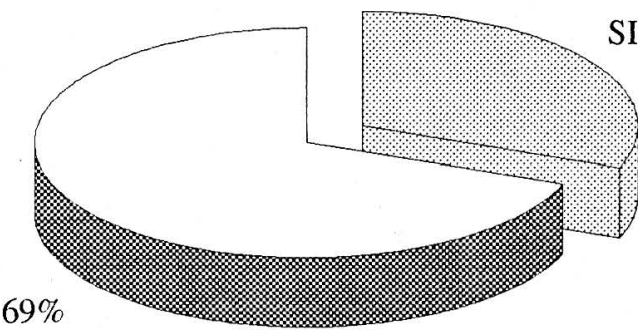


Es importante destacar que de las adolescentes de la presente investigación, el 30.6 presentan una alta exposición a los factores de riesgo para desarrollar el cáncer del cuello uterino.

Tabla 2

ACTITUD FRENTE A LA CITOLOGIA VAGINAL EN ADOLESCENTES ESCOLARES DE MANIZALES

\begin{tabular}{|lrc|}
\hline Actitud & $\mathbf{N}$ & $\boldsymbol{\%}$ \\
\hline Positiva & 106 & 28.3 \\
Indiferente & 175 & 46.7 \\
Negativa & 93 & 25 \\
\hline Total & 374 & 100 \\
\hline
\end{tabular}

Esta Tabla refleja que el mayor porcentaje de los estudiantes encuestados, tienen una actitud indiferente frente a la citología vaginal $(46.7 \%)$.

Figura 2

EDAD DE LA PRIMERA RELACION SEXUAL DE LOS ESCOLARES ADOLESCENTES DE MANIZALES

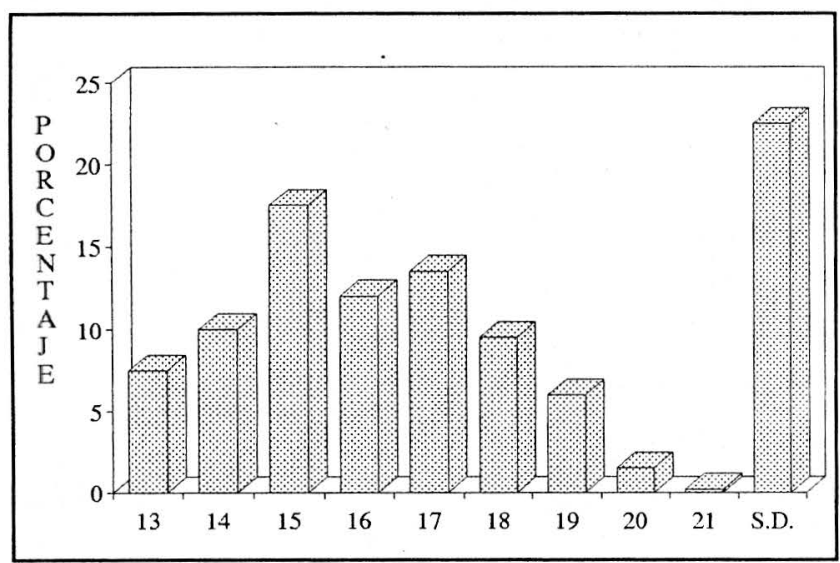

Puede observarse en esta Gráfica que las adolescentes que han tenido relaciones sexuales, el $69,1 \%$ tuvo su primera relación coital entre los 13 y los 18 años.

Tabla 3

ACTIVIDAD SEXUAL DE LAS ADOLESCENTES ESCOLARES DE MANIZALES

\begin{tabular}{|lrr|}
\hline Actividad sexual & $\mathbf{N}$ & $\boldsymbol{\%}$ \\
\hline Si & 142 & 38 \\
No & 232 & 62 \\
\hline Total & 374 & 100 \\
\hline
\end{tabular}

En esta tabla se observa que el $38 \%$ de la muestra estudiada ha tenido relaciones sexuales.
Figura 3

NUMERO DE COMPAÑEROS SEXUALES DE
LAS ADOLESCENTES ESCOLARES DE MANIZALES

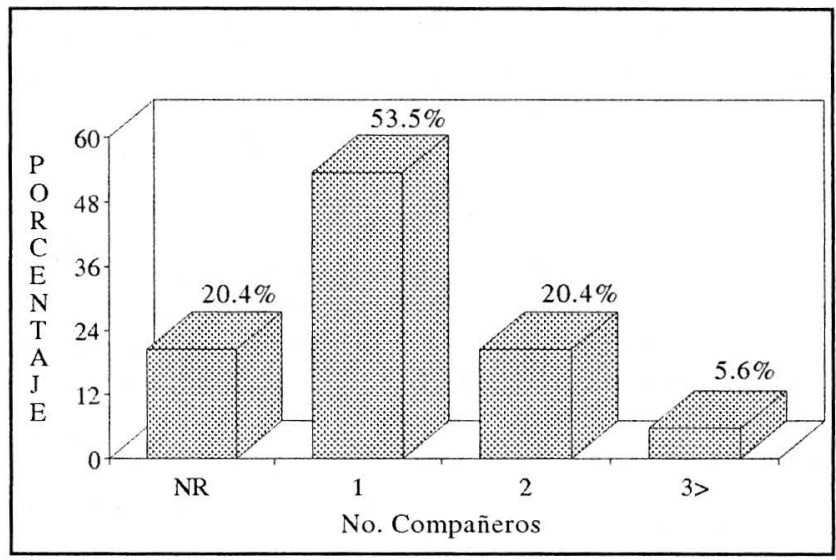

Se observa que el 53,5\% de las adolescentes ha tenido relaciones sexuales solamente con una persona del sexo opuesto y el $20,4 \%$ con 2 personas.

Tabla 4

\section{UTILIZACION METODOS ANTICONCEPTIVOS EN LAS ADOLESCENTES ESCOLARES DE MANIZALES}

\begin{tabular}{|c|c|c|c|c|c|}
\hline Utilizac. Método & $\mathbf{N}$ & $\%$ & Método & $\mathbf{N}$ & $\%$ \\
\hline \multirow[t]{2}{*}{$\mathrm{Si}$} & 73 & 51.4 & Condón & 32 & 39.5 \\
\hline & & & Pastas & 12 & 13.5 \\
\hline \multirow[t]{2}{*}{ No } & 34 & 24 & D.I.U. & 2 & 2.5 \\
\hline & & & Ritmo & 32 & 39.5 \\
\hline No respondieron & 35 & 24.6 & Otro & 4 & 5.0 \\
\hline Total & 142 & 100 & Total & 82 & 100 \\
\hline
\end{tabular}

Con respecto a la anticoncepción de las adolescentes que han tenido actividad sexual, el 51.4\% emplean métodos para evitar la gestación. Los métodos más utilizados por las adolescentes son el preservativo y el método del ritmo, con un $=X(39.5 \%)$.

Tabla 5

TOMA DE LA CITOLOGIA VAGINAL EN LAS ADOLESCENTES DE MANIZALES, QUE HAN TENIDO RELACIONES SEXUALES

\begin{tabular}{|ccc|}
\hline Citología vaginal & N & \% \\
\hline $\mathrm{Si}$ & 8 & 5.5 \\
$\mathrm{No}$ & 95 & 67 \\
No responde & 39 & 27.5 \\
\hline Total & 142 & 100 \\
\hline
\end{tabular}

En el control citológico vaginal por parte de las adolescentes que han tenido relaciones sexuales no ha 
sido utilizado por un $67 \%$ y solamente el $5.5 \%$ se han sometido a este procedimiento.

Tabla 6

CONOCIMIENTO DE LAS ESTUDIANTES ADOLESCENTES DE MANIZALES SOBRE LOS FACTORES DE RIESGO PARA CA DE CUELLO UTERINO

\begin{tabular}{|lcc|}
\hline & \multicolumn{2}{c|}{$\%$} \\
\hline Factores de riesgo & Si & No \\
\hline Virginidad & 10.7 & 89.3 \\
Drogadicción & 37.5 & 62.5 \\
Sexualidad activa & 43.7 & 56.3 \\
Embarazo & 69.2 & 30.8 \\
Promiscuidad & 64.3 & 35.7 \\
Masturbación & 35.1 & 64.9 \\
Uso de condón & 25.2 & 74.8 \\
Primer coito antes de 18 años & 20.1 & 79.9 \\
E.T.S. & 75.1 & 24.9 \\
Embarazo antes de 18 años & 19.6 & 80.4 \\
D.I.U. & 68.1 & 31.9 \\
Hábito de fumar & 63.8 & 36.2 \\
Anovulatorios orales & 37.8 & 62.2 \\
Ropa interior de lycra & 19.6 & 80.4 \\
Multiparidad & 12.1 & 87.9 \\
Coito con la menstruación & 34.6 & 65.4 \\
Compañero sexual promiscuo & 59.0 & 41.0 \\
\hline
\end{tabular}

Se observa que un porcentaje alto $(75 \%)$ de la muestra investigada, respondio que las enfermedades venéreas son un factor de riesgo para adquirir el cáncer de cérvix. Los porcentajes relativamente altos se refieren a "haber estado en embarazo" y "más de un compañero sexual". Los porcentajes medios corresponden a "compañero sexual promiscuo", "tener relaciones sexuales" y "hábito de fumar". Un porcentaje muy bajo de la muestra investigada considera como factores de riesgo las "relaciones sexuales antes de los 18 años", "embarazo antes de los 18 años" y "tener tres hijos o más".

Estos 3 últimos factores se consideran altamente significativos para desarrollar el cáncer de cuello uterino (Te Linde, 1992).

Aunque el conocimiento de los factores de riesgo, en forma general resultó Bueno, este análisis por factores revela el poco conocimiento de algunos de ellos (relaciones sexuales antes de los 18 años, embarazo antes de los 18 años y tener 3 hijos o más).

Existe una asociación entre el tipo de colegio y el grado de exposición a los factores de riesgo para adquirir cáncer de cuello uterino, siendo esta correlación estadísticamente significativa.

$\mathrm{Al}$ observar la Tabla se puede inferir que existe una tendencia a que las adolescentes pertenecientes a colegios mixtos presenten una mayor exposición que las de colegios femeninos.
Tabla 7

CONOCIMIENTO SOBRE FACTORES DE RIESGO PARA CANCER DE CERVIX Y LA EXPOSICION A LOS MISMOS

\begin{tabular}{|lrrr|}
\hline & \multicolumn{3}{c|}{ Exposición } \\
Conocimiento & Si & No & Total \\
\hline Excelente & 24 & 51 & 75 \\
Bueno & 73 & 179 & 255 \\
Regular & 6 & 17 & 23 \\
Deficiente & 9 & 12 & 21 \\
\hline Total & 115 & 259 & 374 \\
\hline $\mathrm{x}^{2}=1.84$ & & & \\
$\mathrm{~g} 1=3$ & & & \\
$\mathrm{p}=0.60$ & & & \\
\end{tabular}

Tabla 8

TIPO DE COLEGIO Y EXPOSICION A LOS FACTORES DE RIESGO

\begin{tabular}{|lccc|}
\hline & \multicolumn{3}{c|}{ Tipo de colegio } \\
Exposición & Femenino & Mixto & Total \\
\hline $\mathrm{Si}$ & 39 & 76 & 115 \\
$\mathrm{No}$ & 128 & 131 & 259 \\
\hline Total & 167 & 207 & 374 \\
\hline $\mathrm{x}^{2}$ corregido & 7.75 & $\mathrm{p}=0.0005$ & \\
Corrección & 7.13 & $\mathrm{p}=0.007$ & \\
\end{tabular}

Figura 4

EDAD Y EXPOSICION A LOS FACTORES DE RIESGO PARA ADQUIRIR CANCER DE CUELLO UTERINO EN ADOLESCENTES ESCOLARES DE MANIZALES

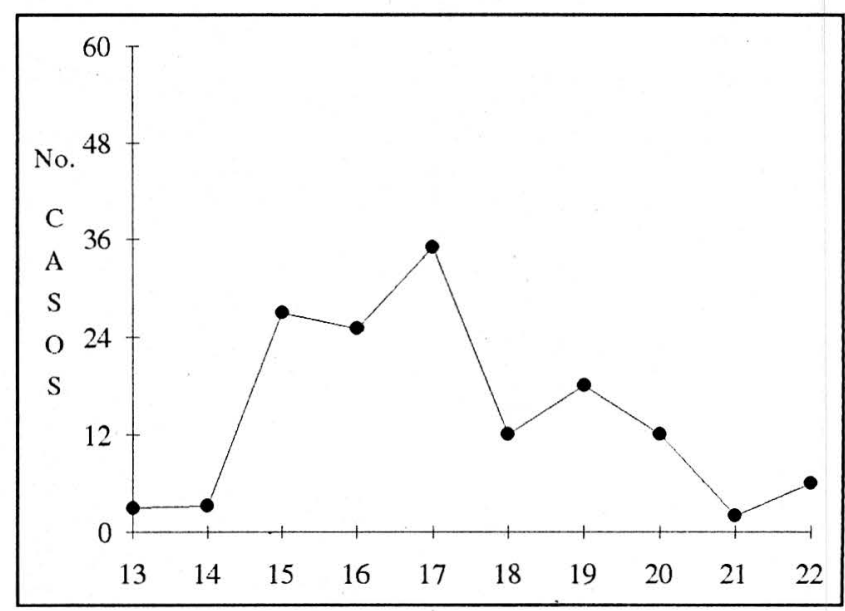

Las adolescentes de 17 años que corresponden al $34,8 \%$ son las que presentan una mayor exposición a los factores de riesgo para adquirir el cáncer del cuello uterino, 5 . 
Tabla 9

\section{CLASIFICACION DEL COLEGIO (PUBLICO Y PRIVADO) Y GRADO DE EXPOSICION A LOS FACTORES DE RIESGO}

\begin{tabular}{|cccc|}
\hline \multicolumn{4}{c|}{ Clasificación Colegio } \\
Exposición & Público & Privado & Total \\
\hline $\mathrm{Si}$ & 98 & 17 & 115 \\
$\mathrm{No}$ & 193 & 66 & 259 \\
\hline Total & 291 & 83 & $374 \cdot$ \\
\hline
\end{tabular}

Existe una asociación estadísticamente significativa entre la clasificación colegio (público-privado) y la exposición a los factores de riesgo para adquirir $\mathrm{Ca}$ de cérvix, puesto que el valor de $\mathrm{p}<0.05$, lo cual sugiere que la clasificación del colegio es un factor que contribuye a la exposición a tales factores, estando más expuestas las adolescentes pertenecientes a las instituciones educativas de carácter público.

\section{Discusión}

Las adolescentes escolares de Manizales tienen en forma general un buen conocimiento acerca de la citología vaginal y de los factores de riesgo para adquirir cáncer de cuello uterino. El $46.7 \%$ tuvo una actitud indiferente hacia la citología vaginal y el $30.6 \%$ están expuestas a tales factores de riesgo.

El 38\% de la muestra estudiada ha tenido relaciones sexuales y el $69 \%$ de estas adolescentes iniciaron su actividad sexual entre los 13 y 18 años.

Con relación a la utilización de la citología vaginal por parte de las adolescentes que han tenido relaciones sexuales, solamente el $5.5 \%$ se han sometido a este procedimiento, considerado este valor muy preocupante, lo que alertaría a las Entidades responsables de Salud para la promoción de estrategias encaminadas a incrementar el uso de este método diagnóstico.

Aunque el conocimiento de los factores de riesgo, en forma general resultó bueno, este análisis por factores revela el poco conocimiento de algunos de ellos, tales como edad del primer coito y del primer embarazo antes de los 18 años, mientras que la promiscuidad sexual, es considerada en un $64.3 \%$ como factor importante para adquirir cáncer de cuello uterino.

Las adolescentes que presentan una mayor exposición a los factores de riesgo para adquirir cáncer de cuello uterino son las de 17 años, que corresponde al $34.8 \%$ de la población estudiada.

Una buena y oportuna educación sexual del adolescente contribuiría a incrementar las actitudes positivas frente a su comportamiento sexual y lo llevaría a decidir conscientemente su exposición a los factores de riesgo para adquirir cáncer de cuello uterino.

\section{BIBLIOGRAFIA}

1. Ministerio de Salud. Manual de Normas Técnico Administrativas. Detección Precoz del Cáncer del Cuello Uterino. Bogotá: Trazo Ltda. 1988.

2. Servicio de Salud de Caldas. Boletín Epidemiológico abril-diciembre, 1990.

3. Te Linde, Richard y Mattingly, Richard. Ginecología Operatoria. 5 ed. Barcelona: El Ateneo S.A., 1980.

4. Rubin, Philip. Oncología Clínica, 5 ed. Nueva York: Universidad de Rochester, 1978.

5. Disaia J., Creasman William T. Clinical Ginecologic Oncology, 2 ed. Toronto: St. Louis, 1984.

6. Ferenczy Alex. The biologic for annual cervical cancer screening. Obstetrics and Ginecology. October, 1985.

7. Balaguero Llado, L. Oncología Ginecológica. Barcelona: Toray S.A., 1980.

8. Corporación Centro Regional de Población. Fuentes de Información de los estudiantes en el campo de sexualidad humana. Bogotá, Vol. 9, marzo 1979.
9. Botero J., Jubiz A., Henao G. Obstetricia y Ginecología, 3 ed. Cali: Carvajal S.A., 1985.

10. El Tiempo. Sexo. 1992; Sección D, pág. 1 D.

11. Useche B., Villegas M., Alzate H. Sexual Behavior of Colombian High School Students. Adolescence, Vol. XXV, No. 98, Summer, 1990.

12. Silber TJ., Kunist N y otros. Manual de Medicina de la Adolescencia. Washington: O.P.S. 1992.

13. Trujillo J. Embarazo juvenil. Memorias XXXI Congreso Nacional de Psiquiatría. Manizales, 1992.

14. Ronderos $\mathrm{M}$. El cáncer del cuello uterino, cáncer del subdesarrollo. A vivir. Minsalud, diciembre 1992.

15. González, Merlo J. Diagnóstico Precoz del Cáncer de Cuello Uterino. Barcelona: Salvat Editores S.A. 1980.

16. Instituto Nacional de Cancerología. Programa Nacional de Cáncer. Fase II. República de Colombia. Ministerio de Salud. Bogotá, oct. 1985 . 